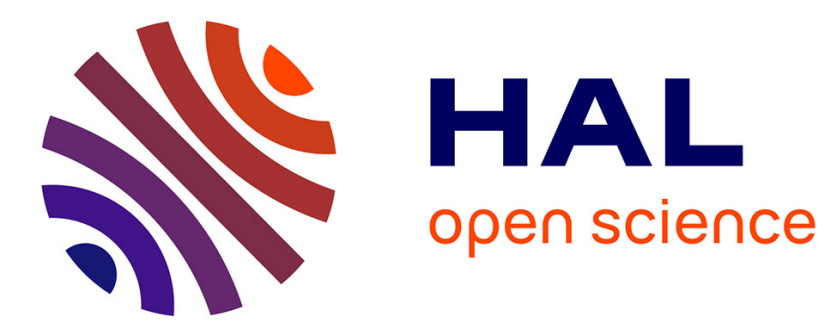

\title{
Le commerce maritime et la croissance européenne au XVIIIe siècle
}

\author{
Guillaume Daudin
}

\section{To cite this version:}

Guillaume Daudin. Le commerce maritime et la croissance européenne au XVIIIe siècle. Christian Buchet, Gérard Le Bouëdec. The Sea in History - The Early Modern World, pp.9-18, 2018, 10.1017/9781782049098.005 . hal-03351996

\section{HAL Id: hal-03351996 https://hal.science/hal-03351996}

Submitted on 22 Sep 2021

HAL is a multi-disciplinary open access archive for the deposit and dissemination of scientific research documents, whether they are published or not. The documents may come from teaching and research institutions in France or abroad, or from public or private research centers.
L'archive ouverte pluridisciplinaire HAL, est destinée au dépôt et à la diffusion de documents scientifiques de niveau recherche, publiés ou non, émanant des établissements d'enseignement et de recherche français ou étrangers, des laboratoires publics ou privés. 


\title{
LE COMMERCE MARITIME ET LA CROISSANCE EUROPÉENNE AU XVIII SIÈCLE
}

\author{
Guillaume Daudin, Université Paris-Dauphine ${ }^{1}$
}

Le commerce international était une partie plus importante des économies européennes autour de 1790 qu'autour de 1830. Il a fallu attendre la grande vague de libéralisation du milieu du $\mathrm{XIX}^{\mathrm{e}}$ siècle pour que le rapport entre le commerce et la taille de l'économie retrouve les taux de la fin du XVIII siècle $^{2}$. L'âge du chemin de fer n'était pas encore arrivé : le commerce au XVIII ${ }^{\mathrm{e}}$ siècle était en grande partie maritime, comme l'était $85 \%$ du commerce français en $1792^{3}$. Le commerce avait atteint ces niveaux importants grâce à une forte croissance. On peut mesurer celle-ci sur des périodes diverses du «petit XVIII $^{\mathrm{e}}$ siècle : plus de $2 \%$ par an pour la France, plus de 1,5\% pour la GrandeBretagne $^{4}, 1 \%$ en termes réels pour l'Allemagne ${ }^{5}, 0,4 \%$ pour les Pays-Bas ${ }^{6}$.

La croissance européenne a été plus rapide dans les régions les plus affectées par le commerce ? D'après les dernières estimations disponibles, la croissance du

\footnotetext{
${ }^{1}$ PSL, Université Paris-Dauphine, LEDa-DIAL UMR IRD 225, F-75016 Paris, France PSL, IRD, DIAL UMR IRD 225, F-75016 Paris, France

SciencesPo, OFCE, F-75007 Paris, France

guillaume.daudin@dauphine.fr

2 O'Rourke K.H., Prados de la Escosura L. et Daudin G., «Trade and Empire », in The Cambridge Economic History of Modern Europe, vol. 1, 1700-1870. BROADBERRY S. et O'Rourke K.H. (dir.), Cambridge, Cambridge University Press, 2010, p. 96-121, tableau 4.5 de la page 106 .

${ }^{3}$ DAUdin G., Commerce et prospérité: la France au XVIIIe siècle. Paris, PUPS, 2005, tableau 12, p. 223.

${ }^{4}$ Ibid., p. 209 et 210.

5 PFISTER U., «The quantitative development of German's international trade during the eighteenth and early nineteenth centuries », in Revue de l'OFCE, 2015, conclusion.

${ }^{6}$ DE VRIES J. et WOUdE A.M. van der, The first modern economy: success, failure, and perseverance of the Dutch economy, 1500-1815. Cambridge, Cambridge University Press, 1997, table 10.13, page 499 .
} 
revenu réel par tête a été entre 1700 et 1800 de 0,4\% en Grande-Bretagne, 0,2 \% en Hollande, 0,1 \% par an en Espagne et en Europe occidentale et - $0,1 \%$ en Italie du Nord ${ }^{7}$. J'ai moi-même proposé $0,6 \%$ de croissance pour la France jusqu'à la Révolution ${ }^{8}$. Tout cela est compatible avec l'hypothèse d'un commerce moteur de la croissance : les pays à la plus forte croissance sont aussi ceux qui on vu leur commerce le plus augmenter. L'examen de l'évolution des salaires réels entre les différents pays européens entre 1300 et 1800 montre aussi que le croissance du commerce intercontinental - forcément maritime - était associé à une croissance plus forte ${ }^{9}$.

Sous-ensemble du commerce, le commerce atlantique a eu un effet particulièrement positif sur la croissance, d'après certains sous condition de bonnes institutions ${ }^{10}$. Cela est moins vrai pour le XVIII ${ }^{\mathrm{e}}$ siècle que pour le XVII ${ }^{\mathrm{e}}$ siècle. En utilisant une version modifiée de la base de données de Bairoch, Batou et Chèvre ${ }^{11}$, on trouve que le taux de croissance des ports pratiquant le commerce atlantique (selon la liste restreinte d'Acemoglu, Johnson et Robinson) n'était que de 0,36\% par an XVIII (à comparer avec 0,32\% pour l'ensemble des villes) alors qu'il était de 0,45\% par an au XVII (à comparer avec 0,19\% pour l'ensemble des villes). La population des villes maritimes en général a augmenté

${ }^{7}$ Bolt J. et ZANDEN J.L., « The Maddison Project: collaborative research on historical national accounts », in The Economic History Review, vol. 67, n 3, 2014, p. 627-651.

8 DAUdin G., Commerce et prospérité, op. cit., p. 28.

9 Allen R.C., « Progress and Poverty in Early Modern Europe », in Economic History Review, vol. $56, \mathrm{n}^{\mathrm{o}} 3,2003$, p. 403-443.

10 Acemoglu D., Johnson S. et Robinson J.A., « The Rise of Europe: Atlantic Trade, Institutional Change and Economic Growth», in American Economic Review, vol. 95, $\mathrm{n}^{\circ} 3$, 2005, p. 546-579 ; DAUDIN G., «Dynamique du commerce atlantique et dynamique de l'intérieur: le cas des ports de Brest à la Corogne», in Les ports du Golfe de Gascogne: De Concarneau à la Corogne (XVe-XXIe siècle). FERnANDEZ A. et MARnot B. (dir.), Paris, PUPS, 2013, p. 33-46 ; DAUDIN G., « Cities of Commerce: how can we test the hypothesis », in Low Countries Journal of Social and Economic History, vol. 11, $\mathrm{n}^{\mathrm{O}} 4$, 2014, p. 103-118

11 Bairoch P., Batou J. et ChÈvre P., La Population des villes européennes: Banque de données et analyse sommaire des résultats 800-1850 / The Population of European Cities: Data Bank and Short Summary of Results. Genève, Libraire Droz, 1988. Pour plus de détails sur la base, voir DAUDIN G., « Cities of Commerce », op. cit. 
de $0.42 \%$ par an entre 1700 et 1800 comparé à $0.27 \%$ par an pour les villes non maritimes ${ }^{12}$.

Le lien entre commerce maritime au sens large et croissance en Europe est donc établi par un faisceau d'indices. La manière dont on peut l'expliquer dépend du type de croissance auquel on s'intéresse: les prémices de la Révolution Industrielle, le progrès technique et l'accumulation du capital ou la croissance smithienne. Dans ces trois cas, l'état actuel des connaissance nous contraint à concentrer notre enquête sur les conséquences du commerce colonial au sens large ${ }^{13}$.

\section{La Révolution Industrielle et la mer}

La Révolution Industrielle britannique a marqué l'avènement de la croissance soutenue, sans diminution ni stagnation de longue durée du produit, dont nous bénéficions toujours. Elle est d'habitude datée de la deuxième moitié du XVIII ${ }^{\mathrm{e}}$ siècle, période où une vague d'innovations techniques ont mécanisé la production et permis d'utiliser de manière intensive le charbon, transformant ainsi durablement l'économie britannique avant de s'étendre au reste de l'Europe.

Cet événement fondateur de l'économie contemporaine est l'un des plus étudiés de l'histoire économique du monde. De très nombreuses explications en ont été données : il n'est pas possible de résumer ces débats ici. La doxa actuelle est que la demande de produits, et notamment la demande extérieure, n'a pas

\footnotetext{
${ }^{12}$ Les villes sont classes comme maritimes si elles avaient un port pouvant accueillir des navires de mer (y compris de pêche) au XIX ${ }^{\mathrm{e}}$ siècle ou avant. Les ports d'estuaire comme Rouen sont inclus.

${ }^{13}$ À ce propos, une synthèse récente présente des discussions pays par pays intéressantes: Roitman J.V., Pétré-Grenouilleau O. et Emmer P.C. (dir.), A deus ex machina revisited: Atlantic colonial trade and European economic development. Brill, 2006. Voir aussi FindLAY R. et O'Rourke K.H., Power and Plenty: Trade, War, and the World Economy in the Second Millennium. Princeton University Press, 2007 et O'Brien P. et DE LA Escosura L.P., « The costs and benefits of European imperialism from the conquest of Ceuta, 1415, to the Treaty of Lusaka, $1974 »$, in La Revista de Historia Económica, vol. 16, nº 1, 1998.
} 
joué un rôle très important ${ }^{14}$. Ainsi, ni les marchés intérieurs ni les marchés extérieurs français n'étaient moins étendus que les marchés britanniques, et pourtant la Grande Bretagne a connu une mutation beaucoup plus rapide que la France $^{15}$. Les explications seraient plutôt à trouver du côté de l'évolution des sciences et des techniques et, d'après Joel Mokyr, des lumières industrielles centrées sur la Grande-Bretagne ${ }^{16}$. L'activité maritime y a joué un rôle : les navires de l'époque moderne étaient de grands utilisateurs des avancées techniques. Ceux utilisés pour le commerce avec l'Asie, comme les vaisseaux de guerre, étaient particulièrement impressionnants de ce point de vue. Tous nécessitaient le développement d'instruments de navigation de plus en plus avancés. L'histoire du développement d'un instrument de mesure du temps qui reste précis malgré les remous des voyages maritimes est bien connue ${ }^{17}$. L'activité maritime a donc joué un rôle important dans l'épanouissement d'un secteur de production d'instruments de précision. Celui-ci a à la fois fournis des outils utiles pour la «Recherche \& Développement» qui s'est systématisée après la Révolution industrielle, et développé des savoir-faire qui ont pu être réutilisés dans les secteurs de la Révolution Industrielle. De plus, l'ouverture sur l'extérieur et la volonté d'émuler les produits asiatiques ont encouragés la recherche, comme le montre le développement de centre de production européens de porcelaine et de toiles imprimées ${ }^{18}$. Tout cela n'est pas négligeable, mais doit comparé à d'autres sources de transformation techniques et intellectuelles comme la mathématisation des sciences et la systématisation de la

\footnotetext{
${ }^{14}$ Mokyr J., «Demand vs. supply in the industrial revolution », in The Journal of Economic History, vol. 37, $\mathrm{n}^{\mathrm{o}}$ 04, 1977, p. 981-1008 est un des premiers articles à presenter cet argument.

${ }^{15}$ Daudin G., « Domestic trade and market size in late-eighteenth-century France », in Journal of Economic History, vol. 70, n ${ }^{\circ}$ 3, 2010, p. 716-743.

${ }^{16}$ Mokyr J., The Enlightened Economy: Britain and the Industrial Revolution, 1700-1850. Penguin, 2011.

${ }^{17}$ Sobel D., Longitude. The True Story Of A Lone Genius Who Solved The Greatest Scientific Problem Of His Time. Fourth Estate, 1996.

${ }^{18}$ BERG M., «From imitation to invention: creating commodities in eighteenth-century Britain », in The Economic History Review, vol. 55, $\mathrm{n}^{\circ}$ 1, 2002, p. 1-30.
} 
procédure expérimentale à partir du XVII ${ }^{\mathrm{e}}$ siècle. Il est difficile de croire que les lumières industrielles n'auraient pas eu lieu sans le commerce maritime.

Cependant, la Révolution industrielle est intimement liée au développement de l'industrie du coton. Ne dépendait-elle donc pas crucialement d'une plante qui n'était disponible pour l'industrie européene que grâce au commerce maritime avec le Proche Orient et, surtout, les Amériques? De plus, la subsistance de la population en croissance rapide en Grande-Bretagne n'a peutêtre été rendue possible que par les calories apportées par la culture du sucre? Kenneth Pomeranz, qui a renouvelé l'étude comparative de l'Europe et de la Chine au à l'orée de la Révolution Industrielle, voit dans l'exploitation des ressources agricoles américaines, grâce à la main d'œuvre des esclaves africains, la principale source de l'avantage de l'Europe sur la Chine ${ }^{19}$. Cette exploitation n'aurait pas été possible sans commerce maritime.

Si on se place du point de vue de la Grande Bretagne des années 1850, le commerce maritime était indispensable pour fournir son industrie en matières premières et sa population en nourriture ${ }^{20}$. Cependant, 1850 est au-delà de notre période d'intérêt. Les structures de l'économie britannique du XVIII ${ }^{\mathrm{e}}$ siècle n'étaient pas celles du XIX ${ }^{\mathrm{e}}$. La croissance britannique au XVIII ${ }^{\mathrm{e}}$ siècle était d'ailleurs beaucoup moins rapide que ne le suggèrent les transformations du secteur du coton, de la métallurgie et du charbon ${ }^{21}$. Dans son ensemble, elle était moins dépendante du commerce maritime que dans les années 1850. Donc, même si on admet, par hypothèse, que la Révolution industrielle n'aurait pas été possible sans le commerce maritime, la croissance liée à celle-ci se lit plutôt dans les chiffres du XIX $^{\mathrm{e}}$ siècle que dans ceux du XVIII ${ }^{\mathrm{e}}$ siècle. Si l'on veut

\footnotetext{
${ }^{19}$ Pomeranz K., The great divergence: Europe, China, and the making of the modern world economy. Princeton, Princeton University Press, 2000.

${ }^{20}$ Clark, O'Rourke et TAYlor, « The growing dependence of Britain on trade during the Industrial Revolution », in Scandinavian Economic History Review, vol. 62, n 2, 2014, p. $109-136$

${ }^{21}$ CRAFts N.F.R. et HARLEY K.C., « Output Growth and the British Industrial Revolution: A Restatement of the Crafts-Harley View », in Economic History Review, vol. 45, n 4, 1992, p. 703-730
} 
comprendre la croissance du XVIII ${ }^{\mathrm{e}}$ siècle, il ne faut pas le faire en ayant les yeux fixés sur le futur : cette méthode fait courir le risque le l'anachronisme.

\section{Le commerce maritime, le progrès technique et l'accumulation du capital}

Revenons donc à l'économie pré-moderne des pays européens. Quelles étaient les sources de sa croissance? Encore une fois, il ne s'agissait pas de l'économie de la Révolution industrielle : le progrès technique industriel, bien qu'il existait, était modeste et incrémentiel. Ce n'est pas dans ses ressorts qu'il nous faut regarder. Quant au progrès technique agricole, d'une part il semble avoir été beaucoup plus modeste qu'on ne l'a longtemps crû et d'autre part il n'a pas été influencé de manière importante par l'introduction de nouvelles plantes : la pomme de terre et le maïs n'ont pas été décisifs à court terme. Les nouvelles méthodes de culture qui ont permis l'augmentation de la productivité de la terre ne dépendaient pas des cultures du Nouveau Monde ou du commerce maritime. Utiliser plus d'animaux, plus d'engrais, plus de semence, plus de travail dans le cadre du «système de Norfolk» (assolement à quatre cultures : navet, orge, trèfle et blé) était une méthode déjà connue dans certaines régions d'Angleterre et des Pays-Bas à la fin du Moyen-Âge ${ }^{22}$.

L'augmentation de la production par tête venait donc d'ailleurs: l'accumulation des facteurs de production, l'intensification de leur utilisation ou leur meilleure allocation.

De nombreux chercheurs, dont le plus célèbre est Marx, ont présenté les entreprises coloniales de l'Europe moderne comme centrale pour l'accumulation primitive de capital ayant précédé la Révolution industrielle ${ }^{23}$. Cette vision traditionnelle a été reprise par l'école marxiste de l'après-guerre, notamment par Eric Williams qui, avant de devenir premier ministre de Trinidad et Tobago, a

\footnotetext{
${ }^{22}$ ALLEN R.C., Enclosure and the yeoman: the agricultural development of the South Midlands 1450-1850. Oxford, Clarendon Press, 1992.

${ }^{23}$ Marx K., Le Capital: Critique de l'économie politique, Livre premier. Paris, Presses Universitaires de France, 1993.
} 
inclus dans les motivations du mouvement abolitionniste britannique de la fin du XVIII siècle la fin du rôle central de l'esclavage rendue possible par la Révolution Industrielle ${ }^{24}$. Le débat sur le sujet porte à la fois sur le niveau des profits censés permettre l'accumulation et leur masse. Les profits du commerce atlantique, pour autant qu'on puisse les mesurer, étaient bien plus élevés que ceux qui pouvaient être espérés dans l'économie intérieure européenne. Cela est vrai même si on prend en compte le risque lié au expéditions maritimes : en effet, à partir d'un certain niveau d'investissement, celui-ci était résorbé par une importante diversification grâce à la prise de participations par chaque investisseur dans de nombreux navires ${ }^{25}$. Certes, les différents secteurs du commerce maritime attiraient de nombreux acteurs qui réduisaient progressivement les surprofits. Cependant, au cours du XVIII ${ }^{\mathrm{e}}$, les investisseurs français ont exploité toute une série de frontières qui chacune et pendant un temps offrait des occasions d'investissement exceptionnelles. Cela a d'abord été

${ }^{24}$ Williams E.E., Capitalism 83 slavery. UNC Press Books, 1944 ; AmIN S., Accumulation on a World Scale. New-York, Monthly Review Press, 1974 ; FrAnK A.G., World Accumulation, 1492-1789. New-York and London, Monthly Review Press, 1978 ; WalleRsteIn I., The modern World system II: Mercantilism and the Consolidation of the European World Economy 16001750. New-York, Academic Press, 1980. CROUZET F. (dir.), Capital Formation in the Industrial Revolution. London, Methuen, 1972, p. 8 donne une liste des prédécesseurs d'Eric Williams.

${ }^{25}$ Thomas R.P. et Bean R.N., «The Fishers of Men: The Profits of the Slave Trade », in Journal of Economic History, vol. 34, no 4, 1974, p. 885-914 ; Anstey R., « The volume and profitability of the British slave trade, 1761-1807 », in Genovese (eds.), Race and Slavery in the Western Hemisphere: Quantitative Studies, 1975, p. 3-31 ; Richardson D., « Profitability in the Bristol-Liverpool Slave Trade », in Revue Française d'histoire d'Outre-Mer, vol. 62, no 226-227, 1975, p. 301-308; Richardson D., « Profits in the Liverpool Slave Trade: The Accounts of William Davenport, 1757-1784», in Liverpool, the African Slave Trade, and Abolition : essays to illustrate current knowledge and research. Anstey R. et Hair P.E.H. (dir.), Liverpool, Historic Society of Lancashire and Cheshire, 1976, p. 60-90 ; INIKORI J.E., « Market Structures and the Profits of the British African Trade in the Late Eighteenth Century », in Journal of Economic History, vol. 41, $\mathrm{n}^{\circ} 4,1981$, p. 745-776 ; DARITY W.J., « The Number Game and the Profitability of the British Trade in Slaves », in Journal of Economic History, vol. 45 February 98, 25, p. 693-703 ; DAUdiN G., « Comment calculer les profits de la traite?», in Outre-Mer: Revue d'Histoire, vol. 89, $\mathrm{n}^{\mathrm{o}}$ 336-337, 2002, p. 43-62 ; DAUdin G., « Profitability of Slave and Long Distance Trading in Context: The Case of 18th Century France », in Journal of Economic History, vol. 64, $\mathrm{n}^{\mathrm{o}} 1,2004$, p. 144-171. 
le commerce avec les Antilles, le commerce d'esclaves, celui avec l'Inde puis avec la Chine.

Même si l'on accepte l'argument sur le niveau des profits, il reste la difficulté liée à leur masse. Les activités liées au commerce colonial ou même maritime en général ne mobilisaient que peu de travailleurs, de capitaux et de terres par rapport à la taille de l'économie européenne dans son ensemble. En conséquence même si le niveau des profits était important, la masse des profits n'a pu représenter qu'une petite partie de l'accumulation du capital totale en Europe. Comme l'a écrit de manière mémorable Patrick O'Brien, les apports en capital de la périphérie n'ont été que périphériques pour le développement de l'Europe $^{26}$. La taille du commerce maritime est d'ailleurs trompeuse à cet égard : il faut effet prendre en compte seulement les revenus en excédent de ce qui aurait été produit par les mêmes facteurs de production utilisés dans l'économie nationale. Mes calculs dans le cas français sont rapportés dans le Tableau 1. Ils indiquent qu'à la fin des années 1780, le revenu total de l'économie française aurait été plus faible de 1.5 à $2 \%$ si le capital et le travail qui étaient investis dans le secteur intercontinental - c'est à dire la partie la mieux connue de l'économie maritime - avaient été rapatriés dans l'économie domestique. Le capital aurait plus souffert de ce rapatriement que le travail. Peut-être est-ce que l'épargne aurait été affectée de manière disproportionnée.

${ }^{26}$ O'Brien P., « European Economic Development: The Contribution of the Periphery », in Economic History Review, XXXV, n ${ }^{0}$ 1, 1982, p. 1-18. Voir aussi SHERIDAN R.B., « The Wealth of Jamaica in the Eighteenth Century », in Economic History Review, vol. 18, n 2, 1965, p. 293-311 ; CoELho P.R., « The profitability of imperialism: The British experience in the West indies 1768-1772», in Explorations in Economic History, vol. 10, $\mathrm{n}^{\mathrm{o}}$ 3, 1973, p. 253-280; Devine T.M., « The Colonial Trades and Industrial Investment in Scotland, c. 1700-1815 », in Economic History Review, vol. 29, $\mathrm{n}^{\mathrm{0}}$ 1, 1976, p. 1-13Solow B., «Caribbean Slavery and British Growth: the Eric Williams Hypothesis », in Journal of Development Economics, vol. 17, $\mathrm{n}^{\mathrm{o}}$ 1, 1985, p. 99-115 ; ELTIS D. et ENGERMAN S.L., « The Importance of Slavery and the Slave Trade in Industrializing Britain », in Journal of Economic History, vol. 60, $\mathrm{n}^{\mathrm{o}} 1$, 2000, p. 123-144. Pomeranz K., The great divergence: Europe, China, and the making of the modern world economy, op. cit. ; Morgan K., Slavery, Atlantic trade and the British economy, 16601800. Cambridge University Press, 2000 ; EMmer P.C., The Dutch slave trade, 1500-1850. Berghahn Books, 2006. 
Même si on prend cet aspect en compte, en supposant par exemple que l'investissement dans le secteur intercontinental n'aurait pas été possible durant l'ensemble du siècle, la croissance aurait été plus faible de 6 à $7,5 \%$, pour un perte cumulée à la fin des années 1790 de 2 à $3 \%$ du revenu national. Ces chiffres seraient plus importants pour des économies plus ouvertes, comme la Grande-Bretagne ou les Provinces-Unies, mais ils resteraient de toutes les manières modestes.

Tableau 1 : Le rôle statique du secteur intercontinental (cas de la France, fin des années 1780) ${ }^{27}$

\begin{tabular}{|c|c|c|c|c|c|}
\hline & Revenu total & $\begin{array}{c}\text { Revenu du } \\
\text { capital }\end{array}$ & $\begin{array}{c}\text { Revenu de } \\
\text { la terre }\end{array}$ & $\begin{array}{c}\text { Revenu } \\
\text { du travail }\end{array}$ & $\begin{array}{c}\text { Situation } \\
\text { britannique }\end{array}$ \\
\hline $\begin{array}{c}\text { Taille relative du } \\
\text { secteur intercontinental }\end{array}$ & $\begin{array}{c}4 \%-4.75 \% \mathrm{du} \text { PIB } \\
9,5 \%-15 \% \mathrm{de} \text { la } \\
\text { production industrielle }\end{array}$ & $13 \%-15,5 \%$ & $0 \%$ & $2.5 \%-3 \%$ & $\begin{array}{c}7 \%-8 \% \mathrm{du} \text { PIB } \\
23 \%-26 \% \mathrm{de} l \mathrm{a} \\
\text { production industrielle }\end{array}$ \\
\hline $\begin{array}{c}\text { Conséquences nettes de } \\
\text { l'existence des relations } \\
\text { intercontinentales }\end{array}$ & De $+1,5 \%$ à $+2 \%$ & De $+6,5 \%$ à $+8 \%$ & De -3\% à $-2.5 \%$ & $\begin{array}{c}\text { De }+0 \% \text { à } \\
0.5 \%\end{array}$ \\
\hline
\end{tabular}

\section{La croissance smithienne et la mer}

Si on se borne donc aux moteurs principaux de la croissance après la Révolution Industrielle, le progrès technique et l'accumulation directe de capital, le commerce maritime ne peut pas avoir joué un rôle décisif. Cependant la croissance avant la Révolution Industrielle était due plutôt à l'exploitation des gains de l'échange telle que décrite par Adam Smith ${ }^{28}$. La croissance «smithienne», lorsque le contexte institutionnel et les conditions de la demande la permettaient, conduisait à deux phénomènes : d'une part une intensification de l'utilisation des moyens de production et d'autre part leur meilleure allocation entre les différentes activités de manière à maximiser la production.

${ }^{27}$ DAudin G., « Do Frontiers Give or do Frontiers take? The Case of Intercontinental Trade in France at the End of the Ancien Régime », in A Deus Ex Machina Revisited. Atlantic Colonial Activities and European Economic Development. PÉtré-Grenoullleau O., Emmer P. et Roitman J. (dir.), Leiden, Brill, 2006, p. 199-224; DAudin G., «Profits du commerce international et croissance dans la France du XVIIIe siècle », in Revue Économique, vol. 57, $\mathrm{n}^{\mathrm{o}} 3,2006$, p. 605-613.

${ }^{28}$ SMith A., An Inquiry Into the Nature and Causes of the Wealth of Nations. London, Strahan, 1776 
Une région isolée devait produire tout ce dont elle avait besoin pour subvenir à ses besoins, quitte à éparpiller ses capacités productives de manière inefficace. Une région ouverte au commerce - y compris maritime - pouvait s'approvisionner grâce à celui-ci pour certains biens, par exemple les céréales, et développer sa production, par exemple viticole, au delà de ses besoins immédiats. Cette spécialisation avait deux effets positifs. D'une part, elle permettait d'allouer les moyens de production vers des secteurs qui étaient relativement favorisés dans chaque région par les circonstances géographiques ou autre. D'autre part, même en l'absence d'avantage a priori l'augmentation de la spécialisation des travailleurs permettait d'augmenter leur productivité comme dans le célèbre exemple de la fabrique d'épingle de Smith. Ainsi Phillip Hoffman a montré que dans la France de l'époque moderne, l'efficacité de la production agricole dépendait de manière décisive de sa proximité avec les centres urbains de demande ${ }^{29}$.

Le commerce maritime jouait un rôle dans ces phénomènes par la baisse des coûts de l'échange qu'il permettait. Grâce à lui, des marchés lointains pouvaient offrir des débouchés importants à l'agriculture et l'industrie européenne, encourageant donc la spécialisation. L'arrière-pays de Bordeaux fournissait farines minots, pruneaux, etc. les Antilles françaises, encourageant en cela leur production $^{30}$. L'industrie de toiles de Bretagne dépendait en partie des marchés de l'Amérique Espagnole ${ }^{31}$. La croissance industrielle de Nantes dépendait des biens nécessaires au commerce d'esclaves ${ }^{32}$. L'Angleterre connaissait les mêmes phénomènes ${ }^{33}$. Cependant, le jeu des nombres implique que, rapportée à

29 HofFMAN P.T., Growth in a traditional society: the French countryside, 1450-1815. Princeton University Press, 2000.

${ }^{30}$ Butel P., Les Négociants bordelais, l'Europe et les îles au XVIIIe siècle. Paris, Aubier, 1974.

31 TANGUY J., Quand la toile va: l'industrie toilière bretonne du xvie au xviiie siècle. Éditions Apogée, 1994

32 Boulle P., «Slave Trade, Commercial Organization and the Industrial Growth in 18thCentury Nantes. », in Revue française d'histoire d'Outre-Mer, vol. 59, n 214, 1972, p. 70-112.

33 Boulle P.H., «Marchandises de traite et développement industriel dans la France et l'Angleterre du XVIIIe siècle », in Revue française d'histoire d'outre-mer, vol. 62, n 226-227, 
l'ensemble de l'économie, la taille des secteurs répondant à la demande coloniale n'était pas considérable.

Sous certaines hypothèses sur le goût pour la diversité des consommateurs, ce jeu des nombres est plus favorable à l'effet des produits offerts par le commerce maritime et le Nouveau Monde. Le sucre, le tabac, le café, le thé, le cacao, les toiles de coton imprimées, les porcelaines... ont acquis au XVIII ${ }^{\mathrm{e}}$ siècle une place de choix dans la consommation des Européens. Boire son café au lait était devenu à la fin du XVIII $^{\mathrm{e}}$ siècle une manière banale de commencer sa journée pour les ouvriers parisiens. Le café devient aussi important en Allemagne. C'est à cette époque que les Britannique ont commencé à boire du thé. Ces produits, souvent addictifs, avaient aussi pénétré les campagnes les plus reculées, comme celles des Alpes ${ }^{34}$. Ils n'étaient pas très importants en termes de calories, mais ils apportaient des goûts et des sensations nouveaux aux consommateurs européens. Hans-Joachim Voth et Jonathan Hersh ont calculé que l'Anglais moyen aurait été disposé à voir son revenu abaissé de $15 \%$ pour conserver un accès au thé et au sucre en $1850^{35}$. La consommation de biens Américains en Europe au XVIII ${ }^{\mathrm{e}}$ siècle était inférieure à celle de l'Angleterre de 1850. Cependant, le sucre et le thé n'étaient pas les seuls biens de consommation rendus disponibles par le commerce maritime. Il faudrait d'ailleurs aussi inclure les matières premières industrielles, par exemple les teintures. Bref, les nouveaux biens avaient eu sur l'économie européenne un effet

1975, p. 309-330 ; INIKORI J.E., Africans and the Industrial Revolution in England: A study in international trade and economic development. Cambridge University Press, 2002 ; O'BRIEN P.K. et EngERman S.L., « Exports and the Growth of the British Economy from the Glorious Revolution to the Peace of Amiens », in Slavery and the Rise of the Atlantic System, SoLOW B. (dir.), 1991, p. 177-209

34 RADEFF A., Du Café dans le chaudron: économie globale d'Ancien Régime, Suisse Occidentale, Franche-Comté et Savoie. Lausanne, Société d'Histoire de la Suisse Romande, 1996.

${ }^{35}$ Voth H.-J. et HeRSh J., « Sweet Diversity: Colonial Goods and the Rise of European Living Standards after $1492 »$, in CEPR Working Paper, 2009. 
au moins comparable, et peut-être un peu supérieur, à l'accumulation du capital.

L'effet sur le bien-être des consommateurs européen est important car, d'après la thèse de Jan De Vries, l'un des moteurs de la croissance avant la Révolution industrielle était la «révolution industrieuse », c'est à dire l'intensification de l'effort de travail - concomitant d'une baisse du temps réservé aux loisirs - dans les ménages européens ${ }^{36}$. Les biens fournis par le commerce maritime s'inscrivaient en cela dans un ensemble de nouveaux biens qui ont contribué à profondément changer les habitudes de consommation au XVIII ${ }^{\mathrm{e}}$ siècle ${ }^{37}$.

Un autre socle de la croissance smithienne était l'extension des capacités d'échange dans l'économie. Pour que les consommateurs travaillent plus pour accéder à de nouveaux produits offerts par les producteurs ou les commerçants, il fallait que les coûts de l'échange ne soient pas trop élevés. Les nouvelles routes britanniques à péage ne peuvent pas être attribuées au commerce maritime. Mais Daron Acemoglu et ses co-auteurs ont affirmé que cela pouvait être le cas des institutions qui encadraient l'échange ${ }^{38}$. Ils se placent dans le cadre de l'économie de coûts de transaction dont l'importance pour l'histoire économique a été soulignée par Douglass North ${ }^{39}$. Comme l'avaient souligné les observateurs contemporains - dont Voltaire dans la dixième Lettre Philosophique - et comme l'affirme l'historiographie whig britannique, le statut social et politique exceptionnellement élevé des marchands en Angleterre y a

\footnotetext{
${ }^{36}$ DE VRIES J., «The Industrial Revolution and the Industrious Revolution », in Journal of Economic History, vol. 54, $\mathrm{n}^{\mathrm{O}}$ 2, 1994, p. 249-270 ; DE VRIES J., The Industrious Revolution: Consumer Behavior and the Household Economy, 1650 to the Present. Cambridge, Cambridge University Press, 2008.

${ }^{37}$ Roche D., Histoire des choses banales : Naissance de la consommation, XVIIe-XVIIIe siècle. Paris, Fayard, 1997.

${ }^{38}$ Acemoglu D., Johnson S. et Robinson J.A., « Rise of Europe », op. cit.

${ }^{39}$ COASE R.H., «The Nature of the Firm », in Economica, , no 4, 1937, p. 386-405 ; NORTH D.C., Institutions, institutional change and economic performance. Cambridge university press, 1990 ; Williamson O.E., The economic institutions of capitalism. The Free Press, 1985
} 
encouragé la croissance. Cela était en partie dû à la nouvelle donne politique issue de la Glorieuse Révolution et de la puissance que les profits du commerce maritime offraient aux marchands. Grâce à eux, ils auraient été capables d'imposer des bornes étroites à l'arbitraire de l'exécutif qui est vu par cette école comme le principal obstacle institutionnel à la sûreté des droits de propriété, condition nécessaire au développement. Les Provinces-Unies l'auraient précédé sur le même chemin institutionnel. Celui-ci était par contre fermé pour le Portugal et l'Espagne à cause de leurs institutions. L'exécutif y aurait été capable d'y accaparer même les profits du commerce maritime. la France était dans une position particulière.

Je fais partie de ceux qui ont des doutes sur cette thèse, qui sous-estime par exemple à quel point les marchands dans l'Angleterre du XVII ${ }^{\mathrm{e}}$ siècle était un groupe de pression comme les autres qui ont utilisé la Glorieuse Révolution pour renforcer leurs propres privilèges ${ }^{40}$. Toutefois, l'idée que les marchands jouaient un rôle particulier dans la croissance à l'époque moderne et qu'ils ont été renforcés de manière décisive par les profits du commerce maritime me semble raisonnable. Elle a même un parfum marxiste qui indique de manière rassurante qu'elle n'appartient pas à une chapelle idéologique particulière.

J'ai gardé la thèse que j'ai défendue dans mes propres travaux pour la fin, au risque d'en exagérer l'importance ${ }^{41}$. Je pense moi aussi que les marchands, du négociant au colporteur, jouaient un rôle central dans la croissance smithienne au XVIII ${ }^{\mathrm{e}}$ siècle. Par contre, il me semble qu'il faut s'intéresser, au delà des questions institutionnelles, au moyens dont ils disposaient pour assurer la circulations des biens dans l'économie et payer les coûts de transaction: collecter les informations nécessaires à leur activité, se prémunir contre le méfait, et disposer du capital nécessaire. Je ne crois pas que le commerce maritime fournissait ces moyens : en tant que secteur à hauts profits, il avait

\footnotetext{
${ }^{40}$ Zahedien N., The Capital and the Colonies: London and the Atlantic Economy 1660-1700. Cambridge University Press, 2010.

${ }^{41}$ Daudin G., Commerce et prospérité, op. cit.
} 
tendance plutôt à priver l'économie intérieure de talents et de capitaux attirés par ses promesses de gains. Cela se voit dans le fait que les ports français attiraient beaucoup de marchands de l'intérieur en ascension sociale. Par contre, puisqu'il fallait atteindre un certain niveau de prospérité avant de pouvoir participer à cette activité maritime si attirante - notamment pour jouer le jeu de la diversification qui était le moyen le plus efficace pour lutter contre les fortunes de mer - il encourageait les marchands de l'intérieur à étendre leur activité, à ne pas recycler leurs fortunes trop tôt dans la terre et les honneurs, bref à faire leur travail de marchands au mieux, ce qui rendait possible la croissance smithienne.

\section{Conclusion}

Résumons-nous. Les taux de croissance nationaux et les taux de croissance des populations urbaines indiquent bien qu'il y avait un lien entre la croissance et le commerce maritime au XVIII ${ }^{\mathrm{e}}$ siècle. Pour comprendre ce lien, il ne faut pas être obnubilé par la Révolution industrielle dont les effets décisifs sur les taux de croissance datent plutôt du $\mathrm{XIX}^{\mathrm{e}}$ siècle. Le progrès technique et l'accumulation de capital productif ne dépendaient pas de manière cruciale du commerce maritime. Par contre, la croissance smithienne lui était beaucoup plus lié par l'augmentation de la demande, la baisse des coûts de l'échange, les nouveaux biens qu'il offrait, le renforcement de la place des marchands et l'encouragement de leur activité. L'état de nos connaissance n'est malheureusement pas encore assez avancé pour donner une idée, même approximative, de la part de la croissance européenne au XVIII ${ }^{\mathrm{e}}$ siècle qui peut être expliquée par chacun de ces phénomènes. 